\title{
DISCUSSION
}

\section{Kinematic analysis of seismic slope stability with discretisation technique and pseudo-dynamic approach: a new perspective}

\author{
CHANG-BING QIN*, SIAU CHEN CHIAN* and GEORGE GAZETAS $\dagger$
}

\section{Contribution by George Gazetas}

The following comments refer only to the 'pseudo-dynamic approach' aspect of the paper (Qin \& Chian, 2018a); the kinematic analysis of seismic slope stability, which is the main part of the paper, is not addressed here.

An acceleration varying sinusoidally with time is considered as the (input) base excitation. It is assumed to be the result of vertically propagating waves. Both a horizontal component and a vertical component are considered, having the same frequency, $\omega$, but different amplitude, $k_{\mathrm{h}} \boldsymbol{g}$ and $k_{\mathrm{v}} \boldsymbol{g}$ (respectively). The horizontal component is associated with shear $(\mathrm{S})$ waves, the vertical with dilatational $(\mathrm{P})$ waves. There is nothing wrong with this simplified input motion (although the assumption of identical frequency of the vertical with the horizontal excitation is unrealistic).

For this excitation, however, the distribution of accelerations with depth is given by two expressions, equations (12), one for the horizontal and one for the vertical motion. The discussion contributor addresses here the horizontal component only, as correspondingly similar arguments apply to the vertical component as well.

The first of equations (12) can be written as

$$
a_{\mathrm{h}}=A q(y) \times \sin 2 \pi\left(\frac{t}{T}-\frac{y}{\lambda_{\mathrm{S}}}\right)
$$

in which $A=k_{\mathrm{h}} \boldsymbol{g}$ is the amplitude of the base excitation; $y$ is the distance measured from the base up; $T=2 \pi / \omega$ is the period of the base excitation; $\lambda_{\mathrm{S}}=V_{\mathrm{S}} T$ is the wavelength of the $\mathrm{S}$ wave; and $q(y)$ is a linearly increasing function of $y$ with: $q(0)=1$ (at the base), $q(H)=f$ (at the top) where $f$ is a constant $>1$, and the derivative with respect to $y$ at the top is $q^{\prime}(H)=(f-1) / H$.

Unfortunately, this expression (equation (12a)) describes only an incident ('travelling') S wave - not the actual 'standing' wave field that is unavoidably produced from the interference of the incident waves with the waves reflected at the free surface! (At this point the reflections at the inclined face of the slope, which would make things too complicated, are not considered.)

To see why the harmonic expression of equation (12a) cannot stand alone, the equation is rewritten in shorter form as

$$
a_{\mathrm{h}}=A q(y) \sin (\omega t-\kappa y)
$$

in which $\kappa=\omega / \lambda_{\mathrm{S}}$. From this expression the harmonic displacement, obtained by an elementary double integration with respect to time, is

$$
u_{\mathrm{h}}=-\frac{a_{\mathrm{h}}}{\omega^{2}}=-\frac{A}{\omega^{2}} q(y) \sin (\omega t-\kappa y)
$$

\footnotetext{
* Department of Civil and Environmental Engineering, National University of Singapore, Singapore.

$\dagger$ National Technical University of Athens, Athens, Greece.
}

and the shear strain is obtained by differentiation with respect to $y$

$$
\begin{aligned}
\gamma_{y \mathrm{~h}} & =\frac{\partial u_{\mathrm{h}}}{\partial y} \\
& =\frac{A}{\omega^{2}}\left[q(y) \kappa \cos (\omega t-\kappa y)-q^{\prime}(y) \sin (\omega t-\kappa y)\right]
\end{aligned}
$$

Hence at the free surface, the shear stress is

$$
\begin{aligned}
\tau_{y \mathrm{~h}, \text { surface }} & =\frac{G A}{\omega^{2}}\left[q(H) \kappa \cos (\omega t-\kappa H)-q^{\prime}(H) \sin (\omega t-\kappa H)\right] \\
& =\frac{G A}{\omega^{2}}[f \kappa \cos (\omega t-\kappa H)-(f-1) / H \sin (\omega t-\kappa H)]
\end{aligned}
$$

This expression should vanish at all times, as required by the free field

$$
\tau_{\text {yh,surface }} \equiv 0
$$

But it does not. This would have only been possible if the time factors

$$
\cos (\omega t-\kappa y) \text { and } \sin (\omega t-\kappa y)
$$

could both be eliminated from equation (12e) (in conjunction with equation (2d)). Evidently, this is impossible with the above expression. The role of the reflected wave, which was spuriously neglected in equation (12), would have been exactly that: to provide time-dependent factors that would help cancel out the time, $t$, and hence allow the shear stress $\tau_{y \mathrm{~h} \text {,surface }} \equiv 0$ boundary condition to be satisfied.

Therefore, the horizontal part of equation (12) and the phase differences it implies between the motions at various points in the slope is invalid. The same is true for the vertical counterpart of equation (12), due to which the vanishing of the normal stress $\left(\sigma_{y \text {,surface }} \equiv 0\right)$ at the surface cannot be satisfied.

Epilogue. The misunderstanding that the wave motion consists solely of a travelling wave appeared for the first time in a journal publication almost 30 years ago. Unfortunately, it has been picked up and relentlessly reproduced by several researchers studying mainly retaining walls, but also slopes and bearing capacity, under seismic excitation. Thus, it appears that this mistaken wave description has 'propagated' (metaphorically and almost literally) through the soil dynamics geotechnical literature, to this day. It is about time that it be 'abandoned'.

\section{Authors' reply}

The authors would like to thank the discusser for his interest in this paper and his contribution to the topic on seismic slope stability. The authors' responses to the discusser's points raised in the discussion are provided as follows.

The authors agree with the discusser that the simple pseudo-dynamic approach (SPDA) violates the zero-stress 
boundary conditions at the free surface as presented in this paper. As elaborately derived by the discusser, the shear (normal) stress cannot vanish at the slope crest surface. In the study of seismic slope stability discussed in the main paper, the initiative was placed on the application of discretisation-based kinematic analysis, having the capacity to consider non-uniform soil properties, which the conventional upper bound method cannot fulfil. The SPDA as shown in equation (12) in the main paper was to reflect a simple expression of acceleration and was not intended to reflect the most appropriate dynamic earthquake effect in this slope stability problem due to the violation of zero-stress boundary conditions, which the authors are well aware of. Modifications and improvements to the SPDA have been introduced (Qin \& Chian, 2018b) to resolve this and several other shortcomings, while adopting the discretisation technique as discussed in the main paper.

In fact, similar efforts to resolve the shortcomings of the SPDA have been proposed and applied to earth retaining walls (Bellezza, 2015; Pain et al., 2015, 2017; Rajesh \& Choudhury, 2017). Such an approach was established based on horizontal (vertical) displacement of the soil under seismic loading. Horizontal (vertical) acceleration can be derived by differentiating the soil displacement twice with respect to time $t$, as discussed below.

Considering a plane wave propagating vertically in a Kelvin-Voigt medium, the shear stress $(\tau)$ can be expressed by the shear strain $\left(\gamma_{\mathrm{s}}\right)$ - that is, $\tau=\gamma_{\mathrm{s}} G+\mu\left(\partial \gamma_{\mathrm{s}} / \partial t\right)$, where $G$ is the shear modulus and $\mu$ denotes the viscosity.

For stress waves propagating through a visco-elastic medium, the equations of motion can be expressed with horizontal and vertical displacements. Having satisfied the boundary conditions including zero shear stress at the free surface and displacement boundary conditions, the horizontal and vertical displacement can be derived in closed form. For instance, considering a horizontal base shaking with period $T$ (where $T=2 \pi / \varpi$ ) and specific displacement at the slope toe, $u_{\mathrm{hb}}=u_{\mathrm{h} 0} \cos (\varpi t)$, the horizontal displacement is expressed as

$$
\begin{aligned}
u_{\mathrm{hs}}(y, t)= & \frac{u_{\mathrm{h} 0}}{C_{\mathrm{S}}^{2}+S_{\mathrm{S}}^{2}}\left[\left(C_{\mathrm{S}} C_{\mathrm{SZ}}+S_{\mathrm{S}} S_{\mathrm{SZ}}\right) \cos (\varpi t)\right. \\
& \left.+\left(S_{\mathrm{S}} C_{\mathrm{SZ}}-C_{\mathrm{S}} S_{\mathrm{SZ}}\right) \sin (\varpi t)\right]
\end{aligned}
$$

where

$$
\begin{aligned}
& y_{\mathrm{s} 1}=\frac{\varpi h}{V_{\mathrm{s}}}\left[\frac{\sqrt{1+4 \xi^{2}}+1}{2\left(1+4 \xi^{2}\right)}\right]^{1 / 2} \\
& y_{\mathrm{s} 2}=-\frac{\varpi h}{V_{\mathrm{s}}}\left[\frac{\sqrt{1+4 \xi^{2}}-1}{2\left(1+4 \xi^{2}\right)}\right]^{1 / 2} \\
& C_{\mathrm{S}}=\cos \left(y_{\mathrm{s} 1}\right) \cosh \left(y_{\mathrm{s} 2}\right) \\
& S_{\mathrm{S}}=-\sin \left(y_{\mathrm{s} 1}\right) \sinh \left(y_{\mathrm{s} 2}\right) \\
& C_{\mathrm{SZ}}=\cos \left[\frac{y_{\mathrm{s} 1}(H-y)}{h}\right] \cosh \left[\frac{y_{\mathrm{s} 2}(H-y)}{h}\right] \\
& S_{\mathrm{SZ}}=-\sin \left[\frac{y_{\mathrm{s} 1}(H-y)}{h}\right] \sinh \left[\frac{y_{\mathrm{s} 2}(H-y)}{h}\right]
\end{aligned}
$$

Since the discretisation technique is used to generate a kinematically admissible failure mechanism, the above formulas are also expressed in coordinates for consistency, within a coordinate system illustrated in Fig. 1 in the main paper. By double differentiating equation (43) with respect to time $t$, the seismic horizontal acceleration at time $t$ and depth $y$ results in

$$
\begin{aligned}
a_{\mathrm{h}}(y, t)= & \frac{k_{\mathrm{h}} \boldsymbol{g}}{C_{\mathrm{S}}^{2}+S_{\mathrm{S}}^{2}}\left[\left(C_{\mathrm{S}} C_{\mathrm{SZ}}+S_{\mathrm{S}} S_{\mathrm{SZ}}\right) \cos (\varpi t)\right. \\
& \left.+\left(S_{\mathrm{S}} C_{\mathrm{SZ}}-C_{\mathrm{S}} S_{\mathrm{SZ}}\right) \sin (\varpi t)\right]
\end{aligned}
$$

where $k_{\mathrm{h}} \boldsymbol{g}=-\varpi^{2} u_{\mathrm{h} 0} ; k_{\mathrm{h}}$ is the horizontal seismic acceleration coefficient at slope toe.

In a similar manner, with base displacement $u_{\mathrm{vb}}=$ $u_{\mathrm{v} 0} \cos (\varpi t)$, the vertical displacement can be written as

$$
\begin{aligned}
u_{\mathrm{vs}}(y, t)= & \frac{u_{\mathrm{v} 0}}{C_{\mathrm{P}}^{2}+S_{\mathrm{P}}^{2}}\left[\left(C_{\mathrm{P}} C_{\mathrm{PZ}}+S_{\mathrm{P}} S_{\mathrm{PZ}}\right) \cos (\varpi t)\right. \\
& \left.+\left(S_{\mathrm{P}} C_{\mathrm{PZ}}-C_{\mathrm{P}} S_{\mathrm{PZ}}\right) \sin (\varpi t)\right]
\end{aligned}
$$

where

$$
\begin{aligned}
& y_{\mathrm{p} 1}=\frac{\varpi h}{V_{\mathrm{P}}}\left[\frac{\sqrt{1+4 \xi^{2}}+1}{2\left(1+4 \xi^{2}\right)}\right]^{1 / 2} \\
& y_{\mathrm{p} 2}=-\frac{\varpi h}{V_{\mathrm{P}}}\left[\frac{\sqrt{1+4 \xi^{2}}-1}{2\left(1+4 \xi^{2}\right)}\right]^{1 / 2} \\
& C_{\mathrm{P}}=\cos \left(y_{\mathrm{p} 1}\right) \cosh \left(y_{\mathrm{p} 2}\right) \\
& S_{\mathrm{P}}=-\sin \left(y_{\mathrm{p} 1}\right) \sinh \left(y_{\mathrm{p} 2}\right) \\
& C_{\mathrm{PZ}}=\cos \left[\frac{y_{\mathrm{p} 1}(H-y)}{h}\right] \cosh \left[\frac{y_{\mathrm{p} 2}(H-y)}{h}\right] \\
& S_{\mathrm{PZ}}=-\sin \left[\frac{y_{\mathrm{p} 1}(H-y)}{h}\right] \sinh \left[\frac{y_{\mathrm{p} 2}(H-y)}{h}\right]
\end{aligned}
$$

Differentiating equation (48) twice with respect to time, the vertical acceleration gives

$$
\begin{aligned}
a_{\mathrm{v}}(y, t)= & \frac{k_{\mathrm{v}} \boldsymbol{g}}{C_{\mathrm{P}}^{2}+S_{\mathrm{P}}^{2}}\left[\left(C_{\mathrm{P}} C_{\mathrm{PZ}}+S_{\mathrm{P}} S_{\mathrm{PZ}}\right) \cos (\varpi t)\right. \\
& \left.+\left(S_{\mathrm{P}} C_{\mathrm{PZ}}-C_{\mathrm{P}} S_{\mathrm{PZ}}\right) \sin (\varpi t)\right]
\end{aligned}
$$

in which $k_{\mathrm{v}} \boldsymbol{g}=-\varpi^{2} u_{\mathrm{v} 0} ; k_{\mathrm{v}}$ denotes the vertical seismic acceleration coefficient at the slope toe.

The zero-stress boundary condition can be easily verified, following a similar procedure as presented by the discusser. Apart from this, the soil damping ratio can also be considered in the modified pseudo-dynamic. In this case, the amplitude of seismic acceleration changes non-linearly with depth, unlike assuming a constant soil amplification factor in the simple pseudo-dynamic.

\section{Comparison}

A comparison is presented herein to show the discrepancies between the pseudo-dynamic solutions of limit load at the slope crest surface, considering the simple and modified pseudo-dynamic approach (MPDA). As stated earlier, the discretisation-based kinematic analysis is applied to account for complicated seismic inputs, and the normalised solutions of limit surcharge (in terms of $q_{\mathrm{c}} / \gamma H$ ) are sought based on the following basic input parameters: $H=10 \mathrm{~m}, \beta=40^{\circ}$, $c_{\mathrm{h}}=20 \mathrm{kPa}, c_{0}=30 \mathrm{kPa}\left(c_{\text {const }}=25 \mathrm{kPa}\right), \phi_{\mathrm{h}}=15^{\circ}, \phi_{0}=25^{\circ}$ $\left(\phi_{\text {const }}=20^{\circ}\right), \gamma\left(y_{i}\right)=18 \mathrm{kN} / \mathrm{m}^{3}, \mu_{\mathrm{v}}=0.5$ and $m_{0}=0$. In the pseudo-dynamic analysis, the dynamic properties of harmonic waves are characterised by $T=0.3 \mathrm{~s}, \quad V_{\mathrm{s}}=100 \mathrm{~m} / \mathrm{s}$, $V_{\mathrm{p}}=187 \mathrm{~m} / \mathrm{s}$ and $\xi=5 \%$. 


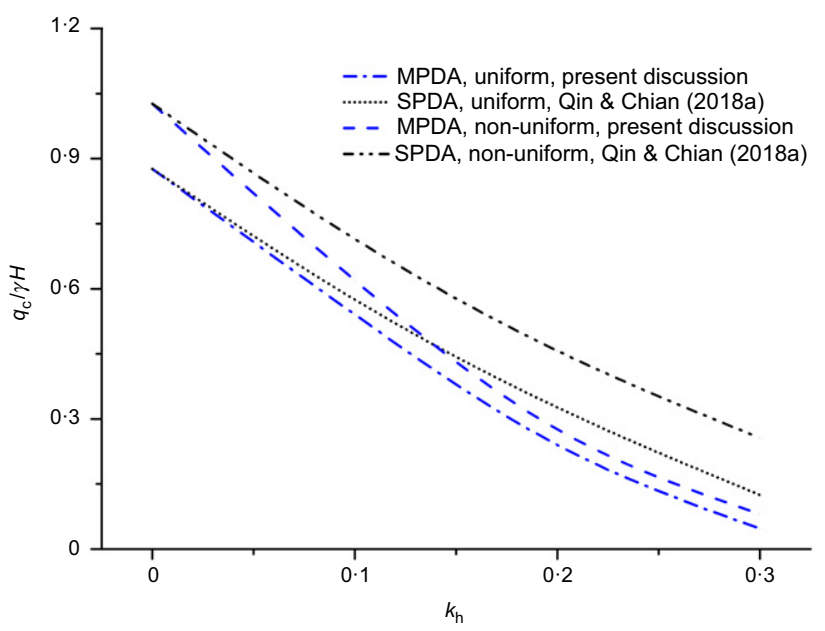

Fig. 21. Comparison of pseudo-dynamic solutions of limit surcharge in uniform and non-uniform soil strata considering different approaches

The first example considers a homogeneous and isotropic (uniform) soil slope where the above varied soil strength parameters are considered with the corresponding average values. The upper bound solutions of limit surcharge are presented in Fig. 21 in this discussion. Apart from identical solutions at no earthquake, an increasing discrepancy is observed with the increase in seismic coefficient $k_{\mathrm{h}}$. As shown in the figure, the solutions obtained from the SPDA are overestimated as compared to the MPDA. Such an overestimation may be attributed to the violation of zero-stress boundary conditions for the simple pseudo-dynamic. In another example, a linearly increasing profile for soil cohesion and friction angle (denoted as non-uniform) is investigated for a normally consolidated soil slope, which is one of the unique capabilities of the proposed discretisation technique presented in the main paper. The comparison results show that the limit surcharge is lower if an average value is assumed in non-uniform soil strata. A higher limit surcharge is again observed in the case of SPDA. It is worthwhile highlighting that the differences between pseudo-dynamic solutions are more pronounced at high $k_{\mathrm{h}}$ values. Therefore, the use of the MPDA combined with the discretisation-based kinematic analysis can potentially optimise seismic slope design, particularly in non-uniform soil strata cases.

The authors trust that readers would find this discretisation technique, as thoroughly discussed in the main paper, to be more versatile and reliable for similar slope stability applications.

\section{NOTATION}

$A$ amplitude of the base excitation ( $\left.=k_{\mathrm{h}} g\right)$

$a_{\mathrm{h}}$ horizontal acceleration
$C_{\mathrm{P}}, C_{\mathrm{S}}, C_{\mathrm{SP}}, \quad$ intermediate variables

$C_{\mathrm{SZ}}, S_{\mathrm{P}}, S_{\mathrm{PZ}}$

$S_{\mathrm{S}}$ and $S_{\mathrm{SZ}}$

$c_{\mathrm{h}}$ cohesion at slope crest surface

$c_{0}$ cohesion at slope toe surface

$f$ soil amplification factor

$G$ shear modulus

g gravitational acceleration

$H$ slope height

$k_{\mathrm{h}}$ horizontal seismic acceleration coefficient at slope toe

$k_{\mathrm{v}} \quad$ vertical seismic acceleration coefficient at slope toe

$q(y)$ linearly increasing function of $y$ with $q(0)=1$ (at the base)

$q_{\mathrm{c}}$ limit surcharge load

$T$ period of the base excitation $(=2 \pi / \omega)$

$t$ time

$u_{\mathrm{h}}$ horizontal displacement

$V_{\mathrm{p}}$ primary wave velocity

$V_{\mathrm{s}}$ shear wave velocity

$y$ distance measured from the base up

$\beta$ slope angle

$\gamma_{\mathrm{s}}$ shear strain

$\gamma_{y \mathrm{~h}}$ shear strain

$\kappa$ intermediate variable $\left(=2 \pi / \lambda_{\mathrm{S}}\right)$

$\lambda_{\mathrm{S}} \quad$ wavelength of the $\mathrm{S}$ wave $\left(=V_{\mathrm{S}} T\right)$

$\mu$ viscosity

$\xi$ damping ratio

$\sigma_{y \text {, surface }}$ normal stress at the free surface

$\tau_{y \mathrm{~h}} \quad$ shear stress

$\begin{array}{ll}\phi_{\mathrm{h}} & \text { friction angle at slope crest surface }\end{array}$

$\phi_{0} \quad$ friction angle at slope toe surface

$\omega$ angular velocity

\section{REFERENCES}

Bellezza, I. (2015). Seismic active soil thrust on walls using a new pseudo-dynamic approach. Geotech. Geol. Engng 33, No. 4, $795-812$.

Pain, A., Choudhury, D. \& Bhattacharyya, S. K. (2015). Seismic stability of retaining wall-soil sliding interaction using modified pseudo-dynamic method. Géotechnique Lett. 5, No. 1, 56-61, https://doi.org/10.1680/geolett.14.00116.

Pain, A., Choudhury, D. \& Bhattacharyya, S. K. (2017). Seismic rotational stability of gravity retaining walls by modified pseudo-dynamic method. Soil Dynamics Earthquake Engng 94, 244-253.

Qin, C. B. \& Chian, S. C. (2018a). Kinematic analysis of seismic slope stability with a discretisation technique and pseudodynamic approach: a new perspective. Géotechnique 68, No. 6, 492-503, https://doi.org/10.1680/jgeot.16.P.200.

Qin, C. B. \& Chian, S. C. (2018b). Seismic bearing capacity of non-uniform soil slopes using discretization-based kinematic analysis considering Rayleigh waves. Soil Dynamics Earthquake Engng. 109, 23-32.

Rajesh, B. G. \& Choudhury, D. (2017). Seismic passive earth resistance in submerged soils using modified pseudo-dynamic method with curved rupture surface. Mar. Georesour. Geotechnol. 35, No. 7, 930-938. 